\title{
Design of Frequency Selective Surfaces for a Gyro-multiplier Output System
}

\author{
Xiang $\mathrm{Li}^{1}$, Xiaoming Liu ${ }^{2}$, Yasir Alfadhl ${ }^{1}$, Xiaodong Chen ${ }^{1}$, Wenlong He ${ }^{3}$, Kevin Ronald ${ }^{3}$, Adrian Cross ${ }^{3}$ \\ 1. School of Electronic Engineering and Computer Science \\ Queen Mary University of London \\ London, England, United Kingdom, E1 4NS \\ 2. School of Electronic Engineering \\ Beijing University of Posts and Telecommunications \\ Beijing, China, 100876 \\ 3. SUPA, Department of Physics \\ University of Strathclyde \\ Glasgow, Scotland, United Kingdom, G1 1XQ
}

\begin{abstract}
This paper presents our on-going work on the development of an output system for a THz gyromultiplier. The beam-wave interaction and frequency multiplication in the gyro-multiplier generate two highorder modes at two distinguished frequencies. To convert the high-order waveguide modes into quasi-Gaussian beams and separate the two frequencies, a quasi-optical mode converter (QMC) and a frequency selective surface (FSS) are combined in the designed output system. The $Q M C$ is used to convert both the high order modes into quasi-Gaussian beams, while the FSS is used to separate the two frequencies. This presentation focuses on the design, fabrication and testing of the required FSS.
\end{abstract}

Keywords: gyro-multiplier; quasi-optical mode converter; frequency selective surfaces.

\section{Introduction}

The operation of a gyro-multiplier is based on the traditional electron cyclotron resonance maser (ECRM) and frequency multiplication $[1,2]$. It is attractive in the terahertz band as the required strength of the external magnetic field is greatly reduced, compared to the traditional low-harmonic gyrotrons. One of such devices has been demonstrated numerically in the University of Strathclyde, showing simultaneous generation of radiation at $342 \mathrm{GHz}$ with the fundamental $\mathrm{TE}_{1,3}$ mode and at 1368 $\mathrm{GHz}$ with the fourth harmonic $\mathrm{TE}_{4,9}$ mode [2]. However, it is known that most of the applications will require single frequency and well collimated wave beam, the generated high-order waveguide modes should be separated and transformed into quasi-Gaussian beams.

In this presentation, our work on the development of an output system for the gyro-multiplier will be described, which consists of a QMC for the transformation [3] and a FSS for the separation [4]. As the designed QMC has been reported elsewhere [5], this paper focuses on the design, fabrication and testing of the required FSS.

\section{System Layout}

Figure 1 shows the schematic view of the output system for the gyro-multiplier. The generated high order waveguide modes are directionally radiated by the Vlasov launcher and adjusted by the subsequent mirrors. The converted quasi-Gaussian beams are then directed to the FSS. The low frequency (LF) content and the high frequency (HF) counterpart are separated by the FSS.

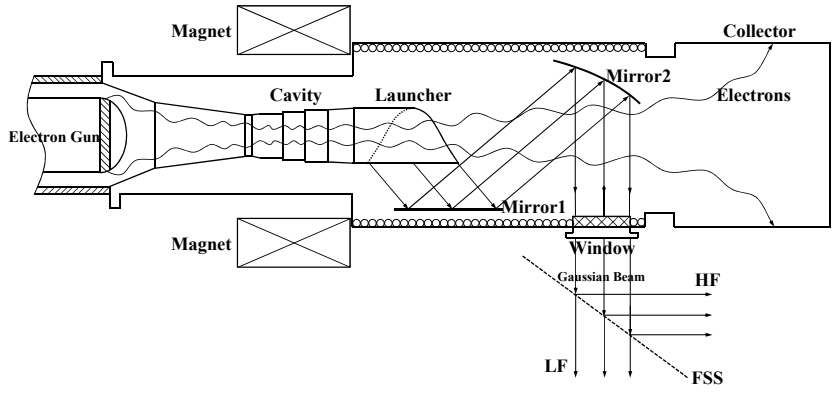

Figure 1. The designed output system configuration.

\section{Simulation of FSS}

With the designed QMC, both modes can be converted into linearly polarized wave beams with energy transfer efficiencies higher than $90 \%$ [5]. To separate the two quasi-Gaussian beams, a FSS is designed by periodic circular perforations of a copper slab, as shown in Figure 2. To avoid the insertion loss at $1368 \mathrm{GHz}$ and lower the fabrication requirement, the designed FSS will reflect the high frequency content while transmitting the low frequency counterpart at $342 \mathrm{GHz}$. The radius of the circular perforation and the distance between two adjacent holes are designed as $0.24 \mathrm{~mm}$ and $0.81 \mathrm{~mm}$, respectively. The thickness of the slab is $0.12 \mathrm{~mm}$.

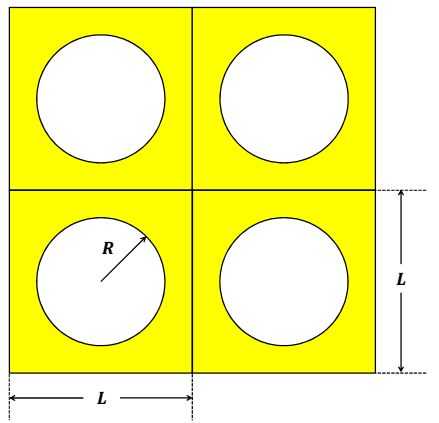

Figure 2. The size of the designed FSS. 
The above design is characterized with CST Microwave Studio [6]. Since the available etching technique provides a fabrication error right below $0.02 \mathrm{~mm}$ regarding the diameter of the holes and the FSS becomes more sensitive to the fabrication error as the incident angle $(\theta)$ increases, the angle of incidence is chosen as $20^{\circ}$. The simulation results in Figure 3(a) and 3(b) demonstrate transmission coefficients $\left(\mathrm{S}_{21}\right)$ of $-0.12 \mathrm{~dB}$ at $342 \mathrm{GHz}$ and that of $26.57 \mathrm{~dB}$ at $1368 \mathrm{GHz}$. This implies that the separation of the two frequencies can be realized with good isolation. The performance of the FSS satisfies the system requirement as $\theta$ varies from $17^{\circ}$ to $23^{\circ}$.

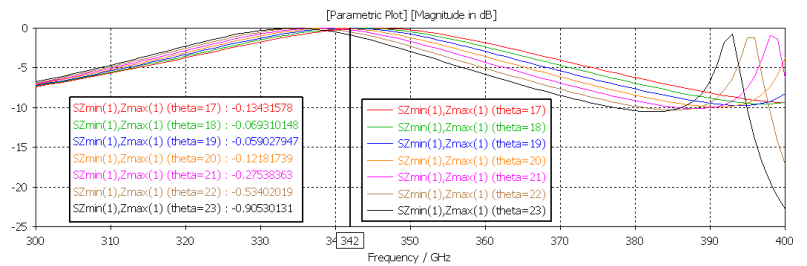

(a)

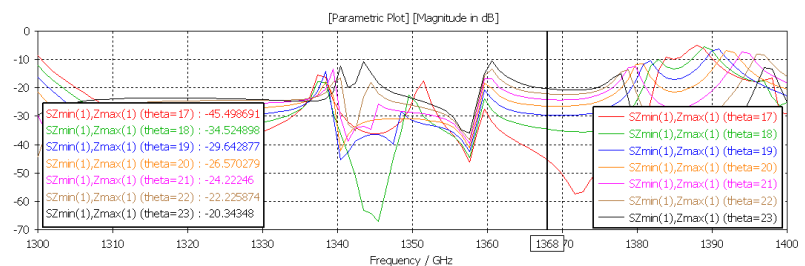

(b)

Figure 3. The $S_{21}$ of the designed FSS at different incident angles. (a) In the lower band; (b) In the higher band;

\section{Prototype Fabrication and Testing}

The sensitivity test regarding the radius of the perforation in Figure 4(a) and 4(b) has shown that the precision provided by the etching technique meets the requirement of the above design.

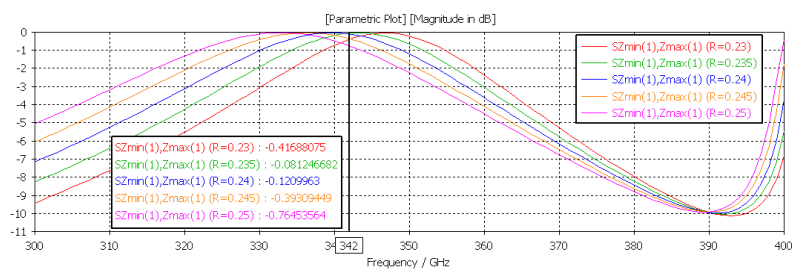

(a)

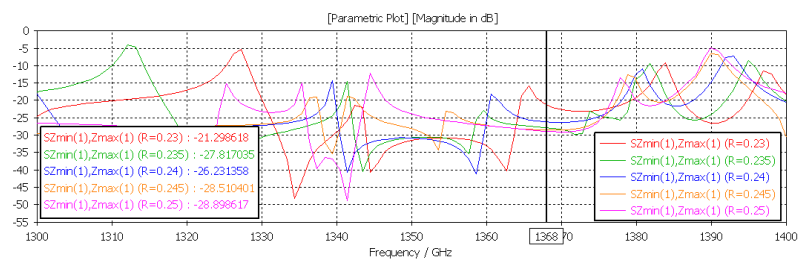

(b)

Figure 4. Sensitivity test regarding the radius of the perforation. (a) In the lower band; (b) In the higher band.
The prototype of the designed FSS has been fabricated as shown in Figure 5. It is formed by an array of $31 \times 31$ perforations. The test of the FSS using a THz-TDS system [7] will be conducted in early 2016 and the results will be presented in the conference.

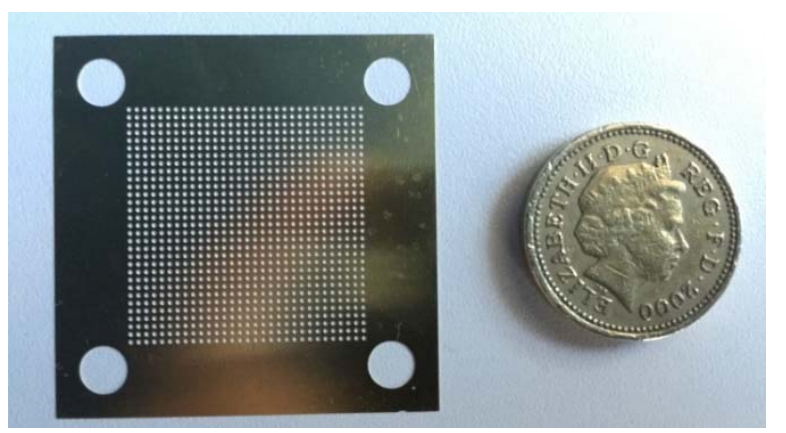

Figure 5. The fabricated FSS sample;

\section{Conclusions}

In this paper, an FSS for the gyro-multiplier output system in the $\mathrm{THz}$ band has been studied. The results have shown that by combining the QMC and the FSS, the dual-frequency output from the gyro-multiplier can be separated and transformed into two quasi-Gaussian beams. The experimental results of the fabricated FSS will be presented in the conference.

\section{Acknowledgements}

This work is supported in part by the National Nature Science Foundation of China under the contract number of 61401031 and the Scientific Research Foundation for the Returned Overseas Chinese Scholars, State Education Ministry.

\section{References}

1. Bandurkin, I. V., et al. "Experimental study of a fourth-harmonic gyro-multiplier." Physics of Plasmas, Vol. 16, 070701, 2009.

2. D. A. Constable, The Numerical and Experimental Investigation of Gyro-multiplier Configurations. PhD Thesis, University of Strathclyde, 2013.

3. S. N. Vlasov, et al. "Geometrical optics of waveguide mode converters." Optics communications 88.4 (1992): 455-463.

4. B. A. Munk, Frequency Selective Surfaces: Theory and Design. New York, US: Wiley, 2000.

5. Xiang Li, et al. "Design of the output system for a gyro-multiplier." $8^{\text {th }}$ Europe/UK-China Workshop on Millimeter Waves and Terahertz Technologies. Cardiff, United Kingdom, September 14-16, 2015.

6. CST Microwave Studio, CST Studio Suite Help Document, 2014.

7. Alexander I Mcintosh, et al. "Terahertz spectroscopy: A powerful new tool for the chemical sciences?" Chemical Society Reviews, Vol 41, Issue 6, 2012 